\title{
Bare-Handed 3D Drawing in Augmented Reality
}

\author{
John J. Dudley ${ }^{1} \quad$ Hendrik Schuff ${ }^{1,2}$ \\ Per Ola Kristensson ${ }^{1}$ \\ ${ }^{1}$ Department of Engineering, University of Cambridge, United Kingdom \\ ${ }^{2}$ Institute for Visualisation and Interactive Systems, University of Stuttgart, Germany
}

\begin{abstract}
Head-mounted augmented reality (AR) enables embodied in situ drawing in three dimensions (3D). We explore 3D drawing interactions based on uninstrumented, unencumbered (bare) hands that preserve the user's ability to freely navigate and interact with the physical environment. We derive three alternative interaction techniques supporting bare-handed drawing in AR from the literature and by analysing several envisaged use cases. The three interaction techniques are evaluated in a controlled user study examining three distinct drawing tasks: planar drawing, path description, and 3D object reconstruction. The results indicate that continuous freehand drawing supports faster line creation than the control point-based alternatives, although with reduced accuracy. User preferences for the different techniques are mixed and vary considerably between the different tasks, highlighting the value of diverse and flexible interactions. The combined effectiveness of these three drawing techniques is illustrated in an example application of 3D AR drawing.
\end{abstract}

\section{ACM Classification Keywords}

H.5.1. Information Interfaces and Presentation (e.g. HCI): Artificial, augmented, and virtual realities.

\section{Author Keywords}

Augmented reality; 3D drawing; interaction.

\section{INTRODUCTION}

Drawings provide a rich means of information exchange. On paper, a rough drawing (sketch) can be constructed quickly, without requiring precision, in order to convey the key features of a concept [4]. While drawing on paper is fast and flexible, markings can only be made in two dimensions. Three dimensional (3D) objects can still be described on paper but these drawings are then projections and unavoidably result in information loss.

It is reasonable to hypothesise that drawing directly in 3D may provide a powerful means of information exchange, without

DIS '18, June 9-13, 2018, , Hong Kong

ACM ISBN 978-1-4503-5198-0/18/06 . .\$15.00

DOI: https : //doi .org/10 . 1145/3196709. 3196737 the deficiencies of conventional 2D projections. However, providing a medium for performing 3D drawings is non-trivialwhat is the 3D equivalent of a drawing pad? Physical 3D media are challenged by occlusion and access constraints.

Digital media presents a more viable alternative. Indeed, recent applications such as Tilt Brush and Gravity Sketch provide functionality for drawing in 3D using virtual reality (VR) and mobile-based augmented reality (AR). These applications provide creative environments for generating $3 \mathrm{D}$ content by removing the constraints of conventional physical media.

What drawing in immersive VR lacks, however, is environmental context. In other words, drawings are produced in a virtual environment that is separated from the physical environment of the user. This is not necessarily a problem for many use cases of 3D drawing but can be detrimental where the physical environment is an important source of reference or inspiration. For example, consider a use case in which an engineer seeks to make a preliminary drawing as part of designing modifications to an existing access platform. To complete this task, the engineer must generate a drawing that references sections of the existing platform whilst also accommodating the spatial constraints imposed by other objects in the space, e.g. pipes, structural columns, etc. AR supports such use cases by preserving environmental context and enables drawings that reference, link to or are inspired by the environment.

In this paper, we explore the provision of 3D drawing capabilities for head-mounted AR delivered through bare-handed interactions (i.e. the user does not hold an input device). Specifically, we explore 3D drawing techniques that do not require any input device other than the AR headset itself. We use a Microsoft HoloLens headset as the experimental platform for our investigation. The examination of bare-handed interaction is motivated by two key concerns. First, there are numerous industrial applications of AR drawing in industries such as construction, manufacturing and logistics. Typically workers in these industries must frequently work with hand tools and in other ways interact with the physical environment. A seamless transition between physical and virtual activities is desirable but there is no input device currently available which supports such interactions without encumbering the user. Second, even if input device interactions are preferable, it is undesirable to render a drawing application completely unusable in circumstances where the user does not have their input device. Such circumstances demand the need for a flexible fallback option when no input device is available.

We evaluate three alternative drawing techniques in a controlled user study. Our design and evaluation of these three 
techniques is a response to the need for diversity in interactions to accommodate different user and use case requirements. These drawing techniques were developed with inspiration from the literature and designed to be complementary. Our study results will later highlight the trade-offs between them. The three interaction techniques are informed by five design principles distilled from the literature and deduced from several envisaged use cases of 3D drawing in AR. We evaluate the three interaction techniques in three distinct tasks abstracted from anticipated use cases: creating a planar drawing, illustrating a motion path, and creating a 3D drawing.

We use multiple metrics, such as completion time, template deviation, path length and perceived workload, for performance evaluation. Additionally, participant preferences and feedback was captured in a summative questionnaire.

In summary, our investigation offers the following contributions to the field of AR interface design:

- Five design principles informing the design of drawing functionality for head-mounted AR as distilled from the literature and deduced from several envisioned use cases.

- An empirical evaluation of alternative drawing techniques in three likely common use cases for 3D drawing in AR.

- A demonstration of the combined use of the three different drawing techniques in a hypothetical 3D drawing scenario.

\section{BACKGROUND AND RELATED WORK}

Ivan Sutherland pioneered the concept of computer-based sketches and drawings with Sketchpad [27]. Sutherland demonstrated both the creative and productive uses of computer-based drawings. Now virtual and augmented reality further extend our ability to generate visual content without the constraints of traditional physical media. The ability to perform 3D drawings in situ represents a fundamentally new capability and a powerful means of information exchange.

Early research into 3D drawing was typically hampered by technological difficulties in supporting the basic visual and interaction capabilities. Clark [7] explored the creation of free-form surfaces in 3D. The apparatus included an input 'wand' with a mechanical wire-based positioning system and an head-mounted display (HMD) (incidentally designed by Sutherland), using mechanical sensors to provide tracking. The user of Clark's system could manipulate the contours of the virtual surface using the wand. Clark observed that, despite the cumbersomeness of the system, the $3 \mathrm{D}$ visualisation and interaction capabilities were superior to traditional 2D techniques.

The work of Butterworth et al. [5] is an early example of a more complete 3D modelling application using an HMD. The system contained several tools aimed at allowing users to produce and connect 3D shapes. Importantly the system provided an undo/redo stack which Butterworth et al. [5] observed encouraged users to make "experimental changes to the model". Responding to users' feedback on the lack of modelling constraints, they propose the use of snap-to functionality on grids and surfaces.
Embodied 3D drawing applications can be delivered without the need for an HMD, as demonstrated by HoloSketch [9] and Surface Drawing [23] which employ stereoscopic 2D displays to construct the illusion that objects are floating in space. Using a similar visual setup, Keefe et al. [15] evaluate several single and two-handed drawing techniques developed for producing 3D curves. CAVE based 3D drawing experiences have also been designed and evaluated $[14,28]$.

The potential for hand-held devices to support drawing in through-the-screen AR has seen recent attention. Hagbi et al. [11] explore in-place drawing for AR games. However, this provides $3 \mathrm{D}$ augmentation of a $2 \mathrm{D}$ sketch rather than true embodied 3D drawing. Window-Shaping [13] is a mobile application in which users can generate virtual content based on a sketch-and-inflate metaphor. The application supports 'projective sketching', which means that users can sketch on detected surfaces to define boundaries and generate new virtual objects. These objects can then be transformed and manipulated in the scene. A key limitation highlighted in this study is the lack of precise control when the user is holding the device and drawing on it at the same time.

Recent improvements in VR hardware at the consumer level, have enabled more research examining fully immersive 3D drawing. Arora et al. [1] investigated the impact of the lack of a physical surface on drawing inaccuracies. They demonstrated that providing additional visual guidance can substantially improve drawing accuracy. McGraw et al. [18] used an HTC Vive VR system with motion controls to allow users to control Hermite splines and create swept surfaces. The system is capable of producing unusual 3D objects with unique aesthetics.

\section{Augmenting Drawing Interactions}

Delivering drawing functionality in digital media is related to the broader problem of selecting and manipulating virtual content. To perform a drawing the user must articulate the point or region in which markings should be generated. In a virtual environment the user is not beholden to conventional means of physical articulation.

An extensive range of manipulation techniques have been explored in the literature (see [21] for a useful taxonomy). In 1996, Poupyrev et al. [20] introduced the 'GoGo' interaction technique. The 'GoGo' interaction method augments the physical reach of the user by providing a virtual extension of the hand. A virtual hand is placed along the vector extending from the chest through the real hand, with depth modulated by a linear scaling below a certain reach threshold and by non-linear scaling above a certain reach threshold. Other variants of the GoGo concept have also been proposed such as 'stretch GoGo' in which regions are used to control growth or shrinking of the arm length, and 'indirect GoGo' in which stretching and retracting the virtual arm is controlled by a 3D mouse [2].

As Bowman and Hodges [3] state, "VEs [Virtual Environments] should enhance the physical, cognitive, and perceptual capabilities of the user, allowing them to do things that are impossible in the real world". This statement echos the proposals of prior work $[1,5,9]$, which highlight the potential of allowing users to independently resize the drawing relative 
to themselves to apply detail at an appropriate scale. This concept is described in a manipulation context as "World-InMiniature" [26]. The ability to enhance the user's capabilities while preserving an embodied interaction experience is a key advantage of drawing in digital media. Leveraging this potential does, however, present a unique design challenge.

The related work highlights a developing understanding of what makes embodied 3D drawing effective and enjoyable. Most research to date, however, has ignored the role AR can play in supporting in situ 3D drawing that references and is inspired by the user's environment. We seek to address this gap by exploring simple and efficient bare-handed interaction techniques that can support embodied 3D drawing in circumstances where mobility and manual engagement with the space are primary concerns.

\section{DESIGNING FOR APPLIED DRAWING WITH AR HMDS}

In the context of this study we focus predominantly on 3D AR drawing as an applied exercise for design, documentation and information exchange but acknowledge its potential as a means of artistic expression. This section briefly explores several envisioned use cases for applied drawing in AR and the common design principles that these imply.

\section{Envisioned Use Cases}

The provision of AR through an HMD enables high mobility and keeps the user's hands free to interact with the physical environment. These two key qualities promote use cases in which overlaid virtual content can be leveraged by a worker who must also navigate through and interact with the space. This kind of work practice is easily imagined in industries such as construction, manufacturing and logistics. The ability to quickly generate 3D drawings in situ could have many uses, e.g. documenting an issue, designing a modification to a space, and/or describing a workflow for training purposes.

In situ markup of virtual-physical models with identified issues or desired features is a high value use case of AR drawing in the construction industry. It is desirable to minimise the information loss and maximise information gain as details pass between site visits, issue tracking systems and building information models. We also envisage significant value in AR drawing as a means to unify the preliminary and detailed design process in various in situ applications. For example, a well-designed AR drawing application for use by cabinet or curtain makers could streamline the process of provisional measurement for quotation and detailed design for installation. Provisional client feedback could also be obtained in situ.

Lastly, drawings may be used as part of training delivered with an AR HMD. Allowing trainers to quickly and easily generate training content in situ provides a low overhead means to induct new workers. 3D drawings may be helpful in showing where objects should be placed, what sequences should be followed, and/or what built products should look like.

\section{Design Principles}

The following design principles are distilled from the literature and deduced from the use cases described above. Their intent is to provide a preliminary set of generalised guidance for the designer of 3D drawing applications built for head-mounted AR. They also help to contextualise the design decisions related to the drawing interactions proposed in this paper.

\section{Minimal Viable Markings}

Many uses of embodied AR drawing are likely to stem from momentary observations and encounters in the physical environment. Among many possible uses, the need to describe an identified issue and the need to document a design change inspired by the space are two obvious reasons for generating a 3D drawing. An application designed for this purpose should thus allow users to quickly and easily complete a drawing before the environment changes or other more pressing tasks dominate. The principle of Minimal Viable Markings suggests the provision of a low overhead means of drawing requiring limited effort and time, even when more precise but slower interactions are available. This design principle also reflects the dual purpose of some forms of sketching in that the act of drawing not only produces a visual artifact but it may also help the user document and respond to their thoughts and observations $[10,8]$. The media and tools for drawing should thus allow the user to quickly respond to these potentially fleeting moments of inspiration.

\section{Promote Spatial Awareness}

Current AR HMDs provide relatively small display regions resulting in a narrow effective virtual field-of-view (FOV). The inability to see virtual content in the periphery challenges people's spatial memory. As drawings extend over larger areas, this can become particularly problematic. Visual aids can help users maintain their memory of the position of their virtual elements relative to their current position. 3D grids and snap-to behaviour for $3 \mathrm{D}$ grids and planes have been proposed in the literature [1, 5, 9, 14]. However, Arora et al.[1] caution that there can be a delicate balance between accuracy and destruction of visual aesthetics. An alternative to the provision of visual aids is careful design of interactions that help preserve user spatial awareness. For example, some interactions may better decouple body movement and environment inspection from the act of drawing.

\section{Exploit the Lack of Physical Constraints}

The transferal of sketching from the physical to the virtual world removes many physical constraints. This can be exploited in AR without necessarily severing the contextual association with the physical space. For example, the concept of relative growing or shrinking of virtual objects has demonstrated utility in allowing users to apply detail at appropriate scale $[1,5,9]$. The virtual hand and cursor techniques discussed previously are also examples of interaction metaphors that Exploit the Lack of Physical Constraints. It is easy to imagine from the use cases described that drawings may need to be completed in circumstances where physical access is challenging (see Figure 13 for an example). Augmenting the capabilities of the user may help address such challenges.

\section{Modal Flexibility}

This design principle is a reflection of diversity in user preferences and use cases when it comes to 3D drawing. We hypothesise that different drawing techniques will be preferred in different usage scenarios. This suggests that there is value 

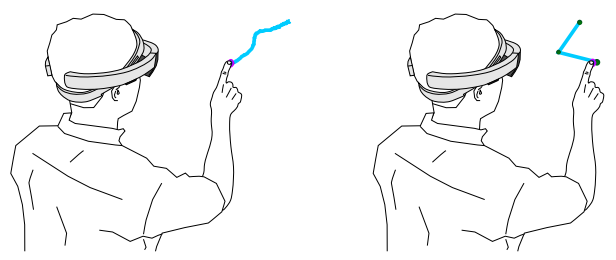

Figure 1. The Freehand (left) and Tapline (right) techniques.

in providing a correspondingly diverse range of drawing techniques which can complement each other. Importantly, there should be a high degree of fluidity when it comes to transitioning between input modes. The virtual manipulation literature has seen a variety of hybrid methods proven effective [2].

\section{Rapid Revision}

This design principle is an extension of the theory of direct manipulation [24] and an application of the golden rule "permit easy reversal of actions" [25] (pp. 71). Butterworth et al. [5] observed the value of providing an undo/redo stack in encouraging experimental behaviour. The need to provide rapid undo and revision functionality is particularly important in circumstances, such as encountered in our system, where the means of controlling the drawing cursor is noisy due to the difficulty of tracking bare hands. Furthermore, Deering [9] observes that fine motor control is harder in 3D than in 2D and Arora et al. [1] highlight that freeform drawing can be inaccurate in 3D. Therefore, embodied 3D drawing is particularly likely to require rapid means of correcting erroneous input.

\section{DRAWING TECHNIQUES}

In response to the anticipated need for quick and simple methods of generating $3 \mathrm{D}$ drawings in $\mathrm{AR}$, we propose three alternative drawing techniques based on bare-handed interactions. These techniques leverage only the egocentric sensing capabilities of the AR headset, leaving the hands free to interact with the physical environment.

The Microsoft HoloLens supports coarse hand tracking only. Only the hand position (a point approximating the centre of the back of the hand) is reported. No other joint or articulation information is available. To deliver our bare-handed drawing techniques, we display a cursor that is directly controlled by hand movement. This cursor (subsequently referred to as the index cursor) is placed at a point approximating the tip of the index finger when the hand is placed in a neutral position with the index finger raised. To reiterate, since no joint or articulation information is available, the index cursor will remain in the same location if the hand/finger articulation changes but the hand position does not. The index cursor is thus a coarse approximation for the hand position but as a direct control scheme, is easily assimilated by the user. The absolute error characteristics of the device's hand tracking are not reported in the literature. Each drawing technique described is, however, subject to the same inaccuracies in hand tracking and the results reflect the summative performance incorporating device induced noise.

By default, the Microsoft HoloLens supports recognition of the air-tap gesture. The air-tap gesture is performed by placing the hand in the neutral position (index finger raised but all other fingers in clench), then bending the index finger at the base knuckle down and raising it again. The fact that the index cursor is not affected by finger articulation changes makes it viable to maintain relatively accurate cursor control while performing finger based gestures. The air-tap gesture is used in all the techniques to trigger line actions. Lack of a secondary default gesture lead us to use double air-tap as an alternative action trigger. The double air-tap is implemented simply by applying a $500 \mathrm{~ms}$ threshold on the time difference between sequential air-tap events. Future iterations of the HoloLens are likely to expose a broader suite of hand gestures. A more complete drawing application could exploit different gestures to deliver more seamless transitions between interaction modes in alignment with the design objective of Modal Flexibility.

The three different drawing techniques are described in detail below. The design of each stems from different combinations of the design principles introduced above.

\section{Freehand}

The Freehand technique allows the user to draw continuously as directed by hand movements. To start a line the user performs the air-tap gesture. The line is drawn continuously at the index cursor position until the user terminates the line by performing a second air-tap. Figure 1 illustrates the Freehand method. The Freehand technique is a response to the design principle of Minimal Viable Markings in that lines can be generated rapidly and easily. The interaction metaphor has strong similarities to finger painting.

\section{Tapline}

The Tapline technique allows the user to draw a line by specifying control points. Tapline seeks to accommodate the design principle of Promote Spatial Awareness in allowing the user to move and look around in between desinating control points. To add a control point, the user directs the index cursor to the desired location and performs an air-tap. This interaction is illustrated in Figure 1. The control points can either be connected by straight lines or by a Catmull-Rom spline [6]. The default setting produces straight polylines but users may optionally switch to splines using the drawing tool belt (introduced below). To terminate the line, the user performs a double air-tap to add a final control point.

\section{GoGo-Tapline}

The GoGo-Tapline represents an extension of the Tapline technique. The user places sequential control points to construct a line as per Tapline. However, the GoGo-Tapline control points are placed at the GoGo-cursor position rather than at the index cursor. The GoGo-cursor is a virtual projection of the index cursor and depends upon the user's current arm extension. If the user's hand is inside a radius of $2 / 3$ arm length around the user's chest, the index cursor and the GoGo-cursor are co-located. Outside this radius, the cursor is extended based on the user's reach as described in [20]. We determine the virtual arm vector, $\mathbf{r}_{\mathbf{v}}$ based on the real arm vector, $\mathbf{r}_{\mathbf{r}}$ (and unit vector $\hat{\mathbf{r}}$ ) according to Equation 1 . Here $\|\cdot\|_{2}$ denotes the Euclidean norm.

$$
\mathbf{r}_{\mathbf{v}}= \begin{cases}\mathbf{r}_{\mathbf{r}} & \text { if }\left\|\mathbf{r}_{\mathbf{r}}\right\|_{2}<D \\ \mathbf{r}_{\mathbf{r}}+k\left(\left\|\mathbf{r}_{\mathbf{r}}\right\|_{2}-D\right)^{2} \hat{\mathbf{r}_{\mathbf{r}}} & \text { otherwise }\end{cases}
$$




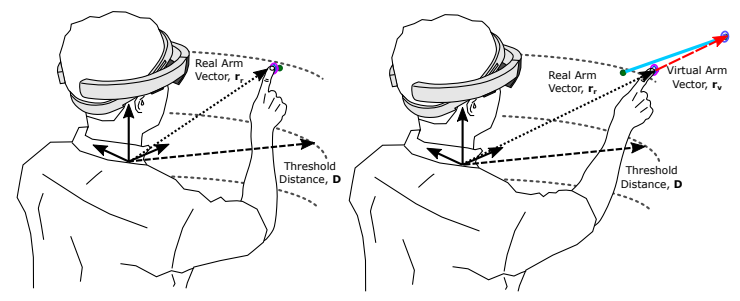

Figure 2. The GoGo-Tapline technique.

Poupyrev et al. [20] selected the scaling coefficient, $k \in[0,1]$ and found good performance choosing a threshold distance, $D$ of $2 / 3$ of the user's arm length. In the implementation presented in this paper, we use $k=1 / 6$ and also choose $D$ to be $2 / 3$ of the distance from the shoulder joint to the middle of the right hand to be consistent with Poupyrev et al. [20]. The GoGo-Tapline drawing technique is illustrated in Figure 2. Reflecting the design principle of Exploit the Lack of Physical Constraints, GoGo-Tapline augments the user's ability to draw lines at distance and without moving location.

\section{Drawing Tool Belt}

The Tapline and GoGo-Tapline drawing features can additionally be adjusted in a tool belt implemented as a floating panel containing several buttons. The tool belt is placed at waist height and follows the user's position. The tool belt allows the user to switch the line style between straight polyline and spline. The current line can also be terminated by pressing Finish Line instead of performing the double air-tap gesture. In a complete drawing application, a tool belt might similarly be important in promoting Modal Flexibility.

\section{USER STUDY}

User performance and experience was evaluated in a withinsubject experiment with three interaction conditions:

- Freehand: continuous line drawing at index cursor.

- TAPLINE: control points placed at index cursor.

- GoGo-Tapline: control points placed at GoGo-cursor.

We recruited 18 participants (3 female, 15 male) with a mean age of $24.6(S D=3.7)$ by convenience sampling. Nine participants reported prior experience with the HoloLens. Participants received a briefing that introduced the headset, described how to use the drawing techniques and described the tasks they must perform. Participants were required to complete a familiarisation task at the start of the experiment and then three drawing tasks in each condition. The familiarisation task and three drawing tasks are described below.

\section{Familiarisation Task}

To reiterate, the user must perform the air-tap gesture to begin a line in FREEHAND or place a control point in TAPLINE and GoGo-TAPLINE. Prior to commencing the main drawing tasks, participants completed a gesture familiarisation task. This task was a simplified version of a circular target acquisition task, with 10 targets presented at incremental angular steps and alternating sides of a circle parallel to the participant's initial view plane. Targets were acquired by navigating

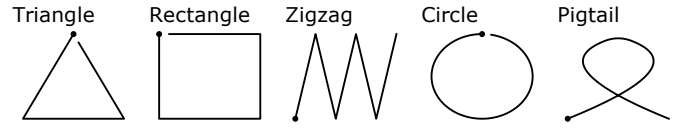

Figure 3. The five 2D templates used in the Follow task.

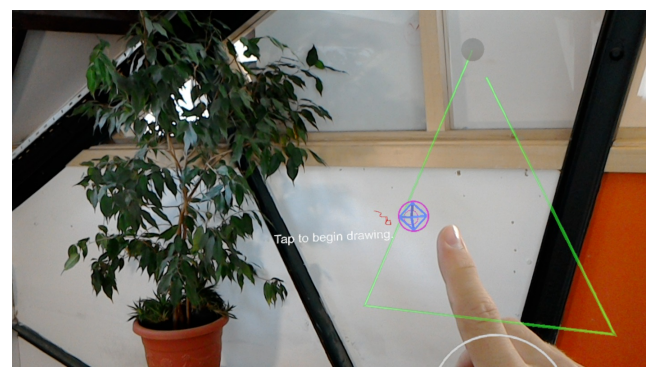

Figure 4. The Triangle as presented in the Follow Template task.

the gaze cursor (a cursor placed at the intersection of the headset forward vector with virtual objects in the scene) to the target and then performing the air-tap gesture. Participants repeated this task, as many times as required, until they could select all ten targets within $15 \mathrm{~s}$. The same task was performed with the double air-tap gesture but with a required completion time of $20 \mathrm{~s}$. This procedure ensured that all participants achieved a base level of proficiency in the air-tap gesture.

\section{Follow Template}

The Follow Template task is derived from a simplified use case in which the user wishes to draw a planar shape at a fixed location in space. Participants were instructed to copy a $2 \mathrm{D}$ planar template, approximately $0.5 \mathrm{~m} \times 0.5 \mathrm{~m}$ in size. All templates were presented in the same location in space. The template remained visible during the task and participants were free to move and position themselves as per their comfort. A standard set of 2D templates were selected from among the shapes used in the $\$ 1$ gesture recognizer [29]. Figure 3 shows the five templates used in this study. Figure 4 shows the Triangle template as seen in the HoloLens by the participant. Participants were asked to draw the template as quickly and as accurately as possible using a single polyline or spline. The Triangle was used as a training task and presented first in all conditions.

\section{Path Indication}

The Path Indication task is derived from an envisaged use case in which the user seeks to indicate a motion path for an object or otherwise associate objects in the space (virtual or physical) by drawing a line. Participants were instructed to draw a path between a Start and Finish node using a single polyline or spline. The drawn path must also pass through multiple mid-point rings. This task is similar to that evaluated by Keefe et al. [15] but at room scale. Five different template paths of varying heights and orientations were constructed. The template path lengths varied between $3.51 \mathrm{~m}$ and $4.41 \mathrm{~m}$ (mean $3.93 \mathrm{~m}$ ). Each unique template path was presented at the same location for all participants. As with the Follow Template task, the first path served as training and was presented first to all participants. This path is shown in Figure 5. 


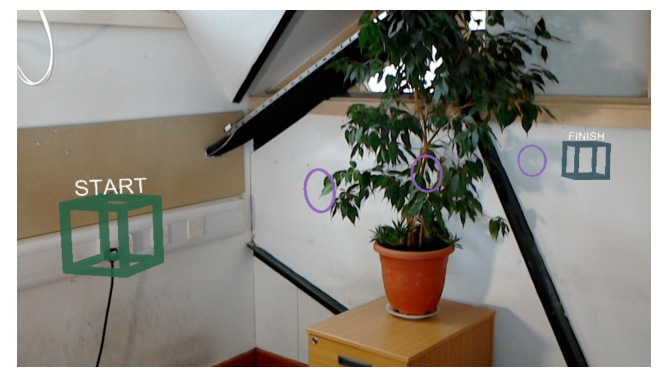

Figure 5. The Start (green), Finish (blue) and Mid-point (magenta) nodes as presented in the Path Indication task.

\section{D Object Recreation}

The 3D Object Recreation task represents an extension of the Follow Template task into 3D. Furthermore, it introduces an element of prompted composition. Participants were instructed to draw lines to recreate a 3D object. The 3D template was presented for $10 \mathrm{~s}$ then the object would disappear (no drawing was permitted while the template was shown). Upon disappearing, participants were required to draw the template from memory. In this task, the participants could use multiple polylines or splines to draw the object. Four different 3D templates were used, see Figure 7. The Cube was presented first as a training task. A view of the Chair as seen through the Hololens is shown in Figure 6. To submit the completed drawing, participants press a Done button in the tool belt.

\section{Procedure}

The order of drawing techniques was assigned using a fully balanced design. Task order was held constant to be consistent with the increasing difficulty of the tasks from easiest (Follow Template) to hardest (3D Object Recreation). The first drawing exercise within each task was presented as a practice exercise. The practice templates in each task were held constant across conditions and participants (i.e. the Triangle, the first unique path, and the Cube). Note that the practice drawings are not included in the subsequent results as the user was encouraged to experiment and ensure they understood the interaction behaviour. The remaining templates for the drawing tasks were randomly shuffled in an effort to minimise confounding ordering effects. The experiment procedure specifically prevented editing or revision behaviour among participants in order to focus on the raw performance capabilities of the drawing techniques. In the Follow and Path Indication tasks, only one line could be drawn and the drawing was automatically submitted upon line termination. Undo or eraser functionality was intentionally removed.

\section{Metrics}

In evaluating the three alternative drawing techniques, we sought to provide resolution in the metrics of performance and user experience. These metrics are summarised below.

\section{Completion Time}

Task completion time was measured based on the elapsed time between the first and last drawing point.

\section{Execution Accuracy}

To measure how well the participants replicate the shapes in the Follow Template task we evaluate two different deviation

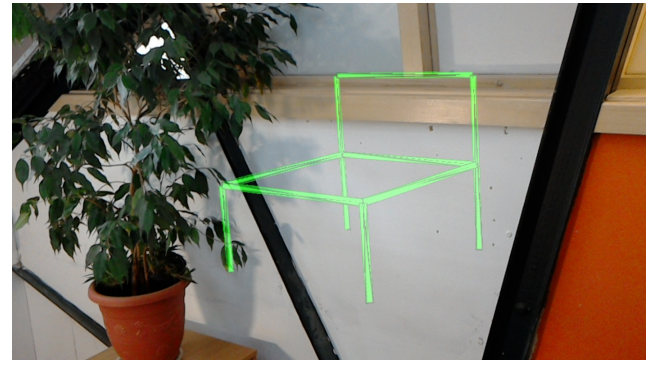

Figure 6. The Chair as presented in the 3D Object Recreation task.

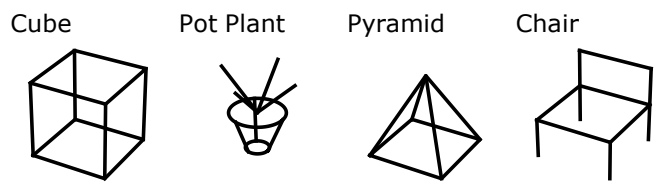

Figure 7. The four 3D Object Recreation templates.

metrics: i) proportional shape matching (psm) described in [17] and Equation 2; and ii) aggregated closest point matching (cpm) described in [15] and Equation 3. Before applying Equations 2 and 3, both polylines are resampled into $N$ equidistant points producing temporally normalised point sequences for the drawing ( $D$ where point $d \in D$ ) and template ( $T$ where point $t \in T)$.

$$
\begin{gathered}
\operatorname{dev}_{p s m}=\frac{1}{N} \sum_{i=1}^{N}\left\|d_{i}-t_{i}\right\|_{2} . \\
\operatorname{dev}_{c p m}=\frac{d_{m}(D, T)+d_{m}(T, D)}{2}, \\
\text { where } d_{m}(D, T)=\frac{1}{N} \sum_{i=1}^{N} \min _{t \in T}\left\|d_{i}-t\right\|_{2} .
\end{gathered}
$$

The $d e v_{p s m}$ metric reflects the average spatial separation between corresponding equidistant sample points in both point sequences. It therefore captures execution direction and large discontinuities but is susceptible to error when drawing behaviour varies inconsistently with time. The $d_{e} v_{c p m}$ metric averages the closest point fit of the drawing to the template and the template to the drawing. It reflects the mean of the shortest distance between points in the two polylines under comparison. A limitation of this metric is that it cannot discriminate between drawings performed in the reverse direction and it regularises discontinuities in the drawn line. As a summative metric of execution quality, however, this is acceptable. For all metric evaluations, the value of $N$ was set at 100 .

A secondary geometric performance metric was used to analyse the drawings completed in the Path Indication and 3D Object Recreation tasks. Normalised line length is the executed drawing length as a percentage of the ideal drawing length for the template.

\section{User Experience}

Before the start of the experiment, participants completed the Simulator Sickness questionnaire [16]. After completion of the three drawing tasks for a given condition, participants 


\begin{tabular}{lccc}
\hline Condition & Time $(s)$ & Dev $_{p s m}(\mathrm{~mm})$ & $\operatorname{Dev}_{\text {cpm }}(\mathrm{mm})$ \\
\hline FREEHAND & $21.6 \pm 11.3$ & $50.6 \pm 27.6$ & $21.8 \pm 15.6$ \\
TAPLINE & $32.5 \pm 17.9$ & $29.3 \pm 16.0$ & $19.0 \pm 10.0$ \\
GoGo-TAPLINE & $40.9 \pm 20.7$ & $40.4 \pm 32.8$ & $22.4 \pm 14.8$ \\
\hline
\end{tabular}

Table 1. Follow Template task mean completion time and mean template deviations. Results show mean \pm 1 standard deviation.

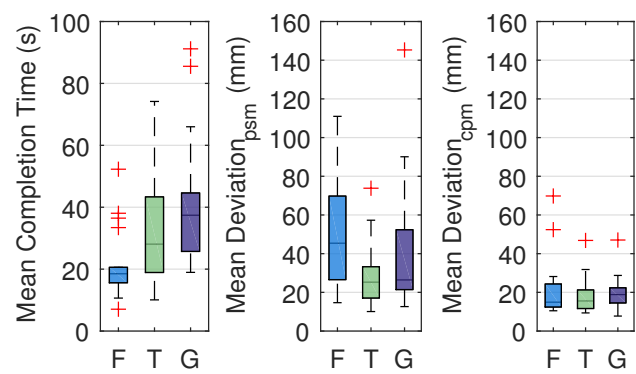

Figure 8. Boxplot of Follow Template task completion time (left), $d e v_{p s m}$ (middle) and $d e v_{c p m}$ (right). The Freehand, Tapline and GoGo-Tapline are abbreviated as $\mathbf{F}$, $\mathbf{T}$ and $\mathbf{G}$ respectively. Red crosses indicate outliers based on the Interquartile Range (IQR) rule, e.g. $q_{3}+1.5 \times\left(q_{3}-q_{1}\right)$.

were instructed to remove the headset and fill out three questionnaires: Simulator Sickness, Flow Short Scale [22] and NASA TLX [12]. At the end of the experiment, participants completed a summative reflection questionnaire that examined their experience of the different drawing techniques and tasks. This questionnaire asked participants to rate their perceived speed, accuracy and comfort with the three drawing techniques on a five point Likert scale. Participants were also asked to provide written comments on their experience with the different techniques and to comment on their experience of the three tasks, independent of the drawing technique used. Finally, participants were asked to indicate their preferred drawing technique for each task and overall.

\section{RESULTS}

The protocol restricting drawings to a single line coupled with user error in performing the control gestures and using the tool belt meant that some drawings were prematurely terminated. Drawings in which participants either stated to have accidentally submitted the drawing or the plotted points show an obvious premature termination were removed from analysis.

Some participants also failed to understand the behaviour of splines when selecting this option to draw the curved templates. For example, several participants drew the Pigtail with control points at the start and end of the template but none in between to capture the curvature. In most cases, this mistake was only made once and participants used the spline behaviour correctly in subsequent drawings. Out of the 72 curved templates executed by participants in Tapline and GoGo-Tapline, 12 are excluded from analysis for this reason.

\section{Follow Template}

The completion time and deviation results for the Follow Task are summarised in Figure 8 and Table 1. The FreEHAND condition produced the quickest completion times, followed

\begin{tabular}{lccc}
\hline Condition & Time $(\mathrm{s})$ & Dev. $(\mathrm{mm})$ & Length $(\%)$ \\
\hline FrEEHAND & $16.0 \pm 9.1$ & $28.7 \pm 10.1$ & $132.5 \pm 32.5$ \\
TAPLINE & $21.2 \pm 9.1$ & $32.1 \pm 32.4$ & $98.3 \pm 3.1$ \\
GoGo-TAPLINE & $30.6 \pm 13.8$ & $37.4 \pm 24.7$ & $103.8 \pm 7.9$
\end{tabular}

Table 2. Path Indication task mean completion time, deviation and normalised line length. Results show mean \pm 1 standard deviation.
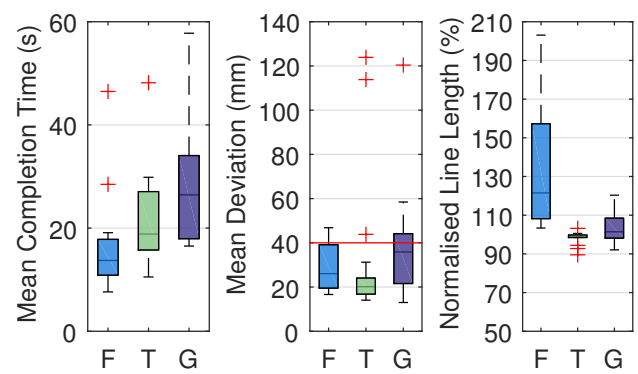

Figure 9. Boxplot of Path Indication task completion time (left), deviation (middle) and normalised line length (right). Red crosses are outliers based on the IQR rule. The red line on the deviation plot (middle) represents the node radius.

by TAPLINE then GoGo-TAPLINE. Repeated measures analysis of variance revealed a significant effect of the drawing technique on completion time $\left(F_{2,17}=14.165, \eta_{p}^{2}=0.455\right.$, $p<0.001)$. A Tukey post-hoc test revealed that the completion times significantly differed between FREEHAND and TAPLINE $(p<0.05)$ as well as FREEHAND and GoGo-TAPLINE $(p<0.001)$. The most accurate drawing technique based on $d e v_{p s m}$ was TAPLINE, followed by GOGo-TAPLINE and then FREEHAND. We found a significant effect of the drawing technique on $\operatorname{dev}_{p s m}$ (RM-ANOVA; $F_{2,17}=3.767, \eta_{p}^{2}=0.181$, $p<0.05$ ). A Tukey post-hoc test showed that the deviation significantly differed between FREEHAND and TAPLINE $(p<0.05)$. The $\operatorname{dev}_{\text {cpm }}$ values listed in Table 1 indicate only marginal differences between the three techniques. The difference observed between $d e v_{p s m}$ and $d e v_{c p m}$ suggests that there are local distortions in FREEHAND that are effectively smoothed by the aggregating closest point metric.

These results indicate that the Freehand technique is an efficient method for executing planar drawings but introduces some minor inaccuracies. In contrast, the Tapline technique is on average $50 \%$ slower but produces significantly more accurate drawings. This is an intuitive result given that the Freehand technique is particularly influenced by the difficulty in fine motor control of the hand in 3D [9] and inaccuracies in the hand tracking compared with the more discrete approach of Tapline. The GoGo-Tapline was marginally slower and less accurate than standard Tapline which we conjecture is due to the additional depth control capability (of no benefit in 2D planar drawing) disrupting participants' use of the technique.

\section{Path Indication}

The Path Indication task required participants to draw a line between a Start and Finish node that also passed through all intermediary nodes. The participant mean completion times, deviations and normalised line lengths are summarised in Table 2 and Figure 9. Note that the mean deviations reported 


\begin{tabular}{lcc}
\hline Condition & Time $(s)$ & Line Length $(\%)$ \\
\hline FREEHAND & $58.3 \pm 23.7$ & $136.4 \pm 30.0$ \\
TAPLINE & $87.7 \pm 38.7$ & $121.3 \pm 29.8$ \\
GoGo-TAPLINE & $93.7 \pm 32.8$ & $141.1 \pm 52.7$ \\
\hline
\end{tabular}

Table 3. 3D Object Recreation task mean completion time and mean normalised line length. Results show mean \pm 1 standard deviation.
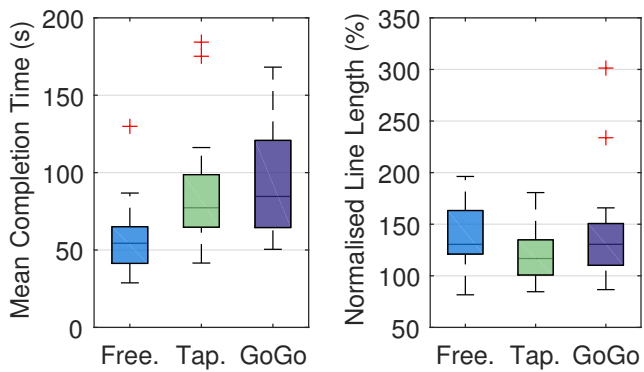

Figure 10. Boxplot of 3D Object Recreation task completion time (left) and normalised line length (right). Red crosses are outliers based on the IQR rule.

here are based only on the closest point deviation from the path nodes. The path nodes had a radius of approximately $40 \mathrm{~mm}$ (show as a red line in Figure 9) and so path points with deviations below this threshold can be considered as having satisfied the task requirements. The normalised line length is based on the line length drawn divided by the shortest possible line length that passes through the centre of every node.

The fastest condition was again FREEHAND followed by TAPLINE and then GoGo-TAPLINE. The effect of drawing technique was significant (RM-ANOVA; $F_{2,17}=12.078$, $\left.\eta_{p}^{2}=0.415, p<0.001\right)$. A Tukey post-hoc test revealed that the completion times significantly differed between FREEHAND and GoGo-TAPLINE $(p<0.001)$ and between TAPLINE and GoGo-TAPLINE $(p<0.01)$.

The mean deviations summarised in Table 2 indicate that all drawing techniques generally produced paths that met the task conditions (within the node radius of $40 \mathrm{~mm}$ ). Figure 9 shows that there were several outliers in TAPLINE that exceeded the node threshold but otherwise line points were closely clustered within acceptable limits. Greater variation and a higher proportion of points exceeding acceptable limits is observable in FREEHAND and GOGO-TAPLINE.

The analysis of normalised line length indicates that significant superfluous drawing is performed when using the FREEHAND technique (RM-ANOVA; $F_{2,17}=18.176, \eta_{p}^{2}=0.517$, $p<0.001)$. A Tukey post hoc test showed that the line lengths in FREEHAND are distinctly elevated from TAPLINE $(p<0.001)$ and GoGo-TAPLINE $(p<0.001)$, which are generally centred around the ideal line length (i.e. 100\%). This behaviour in FREEHAND is also clearly visible in the produced lines, which deviate significantly from the ideal path while still passing close to the node centres. This is in part a consequence of the narrow FOV that users experience due to the small display region of the HoloLens. This makes it difficult for users to perceive the ideal path to the next node. Tapline
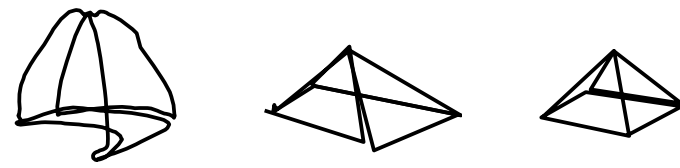

Figure 11. The Pyramid as drawn by one participant in Freehand (left), Tapline (middle), and GoGo-Tapline (right).

and GoGo-Tapline techniques avoid this situation by allowing users to reposition themselves in the space and to look around, independently of executing a drawing.

The results again suggest that Freehand provides a fast but sometimes inaccurate drawing method. It seems reasonable to expect that the Tapline and GoGo-Tapline techniques would be well suited to the Path Indication task given that control points can be precisely placed at the path nodes. However, Tapline and GoGo-Tapline were on average approximately $30 \%$ and $90 \%$ slower respectively than Freehand.

\section{D Object Recreation}

The completion time and normalized line length results for the 3D Object Recreation task are summarised in Table 3 and Figure 10. The fastest drawing technique was FREEHAND followed by TAPLINE and then GoGo-TAPLINE. The condition effect on the completion time proved to be significant (RMANOVA; $\left.F_{2,17}=9.254, \eta_{p}^{2}=0.352, p<0.001\right)$. A Tukey post hoc test revealed that the mean completion times significantly differed between FREEHAND and TAPLINE $(p<0.01)$ and FreeHAND and GoGo-TAPLINE $(p<0.001)$.

The hiding of the 3D template after 10 seconds provided an aspect of prompted composition to the task. As a consequence, however, there is no appropriate template matching metric as participants drew the 3D object from their memory. Instead, we examine the total drawn line length relative to the total template line length. We found a significant effect of the drawing method on line length (RM-ANOVA; $F_{2,17}=3.673$, $\left.\eta_{p}^{2}=0.178, p<0.05\right)$. A Tukey post hoc test revealed that the line length significantly differed between TAPLINE and GoGo-TAPLINE $(p<0.05)$.

These results again favour Freehand in terms of task completion time, with participants completing the object drawings on average approximately $34 \%$ faster than with Tapline. However, Tapline produced objects with total line lengths closest to the ideal template line length.

Figure 11 shows the Pyramid as drawn by one participant using the three different techniques. The Tapline and GoGoTapline Pyramid drawings are considerably more precise and regular than the Freehand drawing. From this example, it is clear that interaction technique can have a significant effect on the resulting aesthetics of a drawing.

\section{Condition Induced Discomfort}

Condition induced discomfort was monitored via the Simulator Sickness Questionnaire [16]. The median responses captured prior to commencing the experiment (PRE-EXPERIMENT) and after each experimental condition are summarised in Table 4. Only the GoGo-TAPLINE condition produced marginal 


\begin{tabular}{lcc}
\hline Condition & Nausea & Oculo-Motor \\
\hline PRE-EXPERIMENT & 0.0 & 1.0 \\
FREEHAND & 0.0 & 1.0 \\
TAPLINE & 0.0 & 1.0 \\
GoGo-TAPLINE & 0.0 & 1.5 \\
\hline
\end{tabular}

Table 4. Median response on the Simulator Sickness questionnaire prior to commencing the experiment and after completion of each condition.

\begin{tabular}{lcc}
\hline Condition & Flow SS & NASA-TLX \\
\hline FREEHAND & 5.4 & 51.2 \\
TAPLINE & 5.7 & 53.0 \\
GOGo-TAPLINE & 5.2 & 67.5 \\
\hline
\end{tabular}

Table 5. Median responses on Flow Short Scale and NASA-TLX surveys.

change in median score on the Oculo-Motor scale. In general, however, all techniques appear to have induced minimal discomfort for users. This is consistent with the qualitative feedback received from users during the study.

\section{Flow and Perceived Workload}

Participant responses to the Flow Short Scale [22] and NASATLX [12] surveys are summarised in Table 5 and Figure 12. These two surveys were completed at the end of each drawing condition. The differences between conditions on the Flow Short Scale responses are marginal with a slight reduction observable in GoGo-TAPLINE. This trend is also repeated in the NASA-TLX workload score where GoGo-TAPLINE is marginally elevated.

\section{Participant Feedback}

At the conclusion of the experiment, participants completed a questionnaire that targeted their experience of the drawing techniques as well as the tasks. The first part of the questionnaire asked participants to rate each of the drawing techniques on a five point Likert scale in response to three statements: 1) The technique made it easy to draw quickly; 2) The technique made it easy to draw accurately; and 3) The technique was comfortable to use. The median statement responses are listed in Table 6. A Friedman test indicated a significant difference in the responses to speed $\left(\chi^{2}(2)=12.18, p<0.01\right)$ and accuracy $\left(\chi^{2}(2)=10.23, p=0.01\right)$ statements. Post hoc analysis with Wilcoxon signed-rank tests was conducted with a Bonferroni correction applied, resulting in a significance level set at $p<0.017$. It revealed significant differences
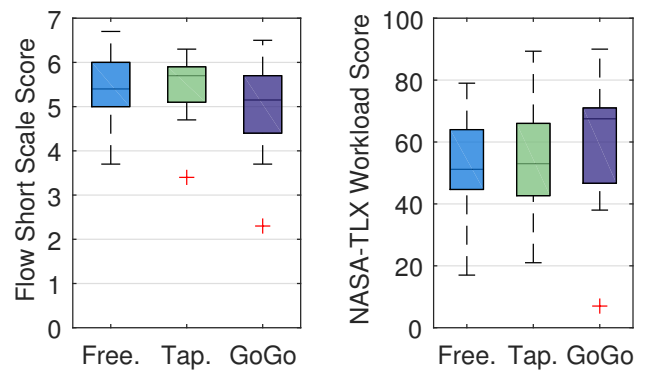

Figure 12. Boxplot of Flow Short Scale responses (left) and NASA-TLX workload (right). Red crosses are outliers based on the IQR rule.

\begin{tabular}{lccc}
\hline Condition & Speed & Accu. & Comf. \\
\hline FREEHAND & 4.5 & 2.0 & 4.0 \\
TAPLINE & 3.0 & 4.0 & 3.0 \\
GoGo-TAPLINE & 2.0 & 2.5 & 3.0 \\
\hline
\end{tabular}

Table 6. Median questionnaire responses assessing perceived speed, accuracy and comfort on a five point Likert scale.

\begin{tabular}{lcccc}
\hline Condition & Follow & Path & 3D Object & Overall \\
\hline FREEHAND & $7(39 \%)$ & $4(22 \%)$ & $5(28 \%)$ & $7(39 \%)$ \\
TAPLINE & $9(50 \%)$ & $7(39 \%)$ & $9(50 \%)$ & $8(44 \%)$ \\
GOGO-TAPLINE & $2(11 \%)$ & $7(39 \%)$ & $4(22 \%)$ & $3(17 \%)$ \\
\hline
\end{tabular}

Table 7. Count of participants' preferences for drawing technique in each task and overall.

between FREEHAND and GoGo-TAPLINE for the speed statement $(Z=-2.173, p<0.05)$ and between FrEEHAND and TAPLINE for the accuracy statement $(Z=-2.560, p<0.01)$.

These results are consistent with the quantitative performance results obtained. The Freehand was fastest in all tasks and was perceived as so by participants. The Tapline technique was the most precise based on the deviation metrics and this was also reflected in participant responses to the accuracy statement. Although not statistically significant based on a Friedman test, the Freehand technique had the highest median comfort rating.

\section{Participant Comments}

Participants were invited in the questionnaire to note any perceived advantages and disadvantages of the three drawing techniques. These qualitative responses were reviewed to identify any commonality. A frequently mentioned advantage of the Freehand technique was the ability to draw fast and easily ("easy, fast"). At the same time, participants criticized that it lacked accuracy ("Hard to keep a straight line."). The Tapline technique received positive feedback regarding accuracy and expenditure of time ("Easiest to place accurately", "Quickness. [...]"). However, participants stated that it is hard to draw curved lines ("curved lines harder to get right"). The GoGo-Tapline was described as comfortable (e.g. "the most convenient, you can just stand and draw") and beneficial in maintaining an overview of the scene while drawing ("The distance allowed by the technique allowed me to see the drawings as a whole [...]"). On the other hand, the depth of the cursor was hard to perceive for multiple participants ("[...] sometimes hard to get the depth correct.").

\section{Preferences}

The final part of the questionnaire invited participants to select their preferred technique for each of the three study tasks and overall. Table 7 summarises the participant preference distribution. The Tapline technique was the most favoured drawing technique for the Follow and the 3D Object Recreation tasks. For the Path Indication task, the Tapline and GoGo-Tapline techniques were equally favoured. Overall, the two roughly evenly favoured techniques were Freehand and Tapline with GoGo-Tapline only preferred by three of 18 participants. These preference responses are interesting to interpret within the context of the performance results. Their 
comparatively even distribution suggests that different users appreciate different capabilities. This highlights the need to support switching between modes in a complete drawing application.

\section{A PRACTICAL EXAMPLE OF 3D AR DRAWING}

As a demonstration of the practical use of the bare-handed drawing techniques presented, one of the authors completed the 3D drawing shown in Figure 13. This drawing was created in situ based on a hypothetical scenario requiring a modification of an existing access platform to create a link to an adjoining building.

The drawing shown in Figure 13 utilises all three drawing techniques. A rapidly executed zigzag (in red) using the Freehand technique indicates the portion of railing to remove. The Tapline technique was used to accurately designate the boundary of this section. The GoGo-Tapline was essential in allowing lines to be drawn on the surface of the adjoining building. The provision of complementary interactions in this application reflects the design principle of Modal Flexibility.

\section{DISCUSSION}

The findings from the user study highlight the influence that task type can have on user performance and experience. Furthermore, the distribution of preferred drawing technique captured by the summative questionnaire demonstrates that different users appreciate different capabilities. Numerous participants highlighted the fact that certain operations were easier with one technique than another. It was in anticipation of this diversity that we sought to test three alternative drawing techniques with distinct advantages and disadvantages.

The Freehand technique yielded the fastest completion times throughout all tasks as well as the highest questionnaire scores regarding speed and comfort. In contrast, the Tapline method was the most precise for the Follow Template and Path Indication tasks where deviation metrics are meaningful. The GoGo-Tapline technique was developed as a demonstration of a drawing-based interaction derived from a virtual hand metaphor and reflects the design principle of Exploit the Lack of Physical Constraints. This was motivated by the belief that allowing users to modulate cursor depth without taking steps may be useful. In practice, the limited use of the virtual extension functionality meant that the GoGo-cursor functioned more like a generic offset cursor [19]. The additional complexity introduced by the interaction method likely confounded the performance results. Nevertheless, the technique was chosen by three of the 18 participants as their overall favourite.

If the tasks had been more demanding in terms of movement or reach, it is likely that the benefits of GoGo-Tapline would have been more clearly revealed. In terms of generating drawings as a general human-computer interaction task, there are several observations that can be made. Participants commented that they were occasionally surprised how distorted the object they were drawing looked when they viewed it from a different angle. This is consistent with observations made by Deering [9] where people had to learn to break 2D interface habits of keeping their head artificially still and instead look around the virtual world. Generally this issue relates to the difficulty

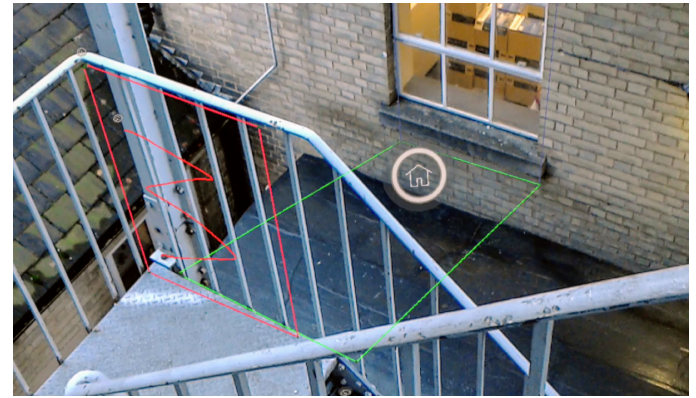

Figure 13. Preliminary design drawing of a hypothetical access modification to join an existing walkway with a neighbouring building. Drawing completed in situ by one of the authors using all three of the drawing techniques. All line elements are spatially fixed to preserve references to the physical space.

of providing fine depth perception of virtual content in AR, as was commented on by several participants in this study.

\section{Limitations and Future Work}

In this paper we focus primarily on the immediate efficacy of the interaction techniques. By necessity, this ignores the common activity in drawing of revision and editing and the design principle of Rapid Revision. Basic line erasing and deletion functionality is provided in the application demonstration in Figure 13 but awaits user validation. Future work will examine the features necessary to provide productive and efficient drawing editing functionality.

As an initial step towards investigating the design principles set out in this paper, the study involved simplified drawing tasks. We suggest an interesting avenue for future work is to target more complicated use cases and contexts to increase the external validity of the findings.

\section{CONCLUSION}

In this paper we presented three interaction techniques supporting bare-handed drawing in AR: Freehand, Tapline and GoGo-Tapline. We studied their performance in a user study with three drawing tasks: Follow Template, Path Indication and 3D Object Recreation. We established that Freehand drawing is a fast, although not necessarily accurate, technique. Tapline, which is based on placing control points to create line segments, achieves a balance between speed and accuracy and was favoured by participants. We also introduced a novel combination of a virtual hand metaphor with the Tapline drawing technique, the GoGo-Tapline technique, which was equally most favoured in the Path Indication task.

The study highlighted the benefit of allowing users to fluidly switch between techniques to exploit the trade-offs between them. The three bare-handed drawing techniques are underpinned by five design principles, which can form a starting point for designers in developing other applications. We strongly believe that $3 \mathrm{D}$ drawing in AR will become a highly effective means of information exchange and will see wide use in both applied and creative applications.

\section{ACKNOWLEDGMENTS}

This work was supported by EPSRC (grant numbers EP/N010558/1 and EP/R004471/1) and the Trimble Fund. 


\section{REFERENCES}

1. Rahul Arora, Rubaiat Habib Kazi, Fraser Anderson, Tovi Grossman, Karan Singh, and George Fitzmaurice. 2017. Experimental Evaluation of Sketching on Surfaces in VR. In Proceedings of the 2017 CHI Conference on Human Factors in Computing Systems (CHI'17). ACM, New York, NY, USA, 5643-5654. DOI : http://dx.doi .org/10.1145/3025453.3025474

2. Doug A. Bowman and Larry F. Hodges. 1997. An Evaluation of Techniques for Grabbing and Manipulating Remote Objects in Immersive Virtual Environments. In Proceedings of the 1997 Symposium on Interactive $3 D$ Graphics (I3D '97). ACM, New York, NY, USA, 35-ff. DOI:http://dx.doi.org/10.1145/253284.253301

3. Doug A. Bowman and Larry F. Hodges. 1999. Formalizing the Design, Evaluation, and Application of Interaction Techniques for Immersive Virtual Environments. Journal of Visual Languages \& Computing 10, 1 (1999), 37-53. DOI: http://dx.doi.org/10.1006/jvlc.1998.0111

4. R. A. Burkhard. 2004. Learning from architects: the difference between knowledge visualization and information visualization. In Proceedings. Eighth International Conference on Information Visualisation, 2004. IV 2004. 519-524. DOI:

http://dx.doi.org/10.1109/IV.2004.1320194

5. Jeff Butterworth, Andrew Davidson, Stephen Hench, and Marc. T. Olano. 1992. 3DM: A Three Dimensional Modeler Using a Head-mounted Display. In Proceedings of the 1992 Symposium on Interactive 3D Graphics (I3D '92). ACM, New York, NY, USA, 135-138. DOI: http://dx.doi.org/10.1145/147156.147182

6. Edwin Catmull and Raphael Rom. 1974. A class of local interpolating splines. In Computer Aided Geometric Design, Robert E. Barnhill and Richard F. Riesenfeld (Eds.). Academic Press, New York, 317-326. DOI : http://dx.doi .org/10.1016/B978-0-12-079050-0.50020-5

7. James H. Clark. 1976. Designing Surfaces in 3-D. Commun. ACM 19, 8 (Aug. 1976), 454-460. DOI: http://dx.doi.org/10.1145/360303.360329

8. Matthew T. Cook and Arvin Agah. 2009. A survey of sketch-based 3-D modeling techniques. Interacting with Computers 21, 3 (2009), 201-211. DOI: http://dx.doi.org/10.1016/j.intcom.2009.05.004

9. Michael F. Deering. 1995. HoloSketch: A Virtual Reality Sketching/Animation Tool. ACM Trans. Comput.-Hum. Interact. 2, 3 (Sept. 1995), 220-238. DOI: http://dx.doi.org/10.1145/210079.210087

10. Gabriela Goldschmidt. 1991. The dialectics of sketching. Creativity Research Journal 4, 2 (1991), 123-143. DOI: http://dx.doi.org/10.1080/10400419109534381

11. Nate Hagbi, Raphael Grasset, Oriel Bergig, Mark Billinghurst, and Jihad El-Sana. 2015. In-Place Sketching for Augmented Reality Games. Computers in Entertainment 12, 3 (2015), 1-18. DOI:

http://dx.doi.org/10.1145/2702109.2633419

12. Sandra G. Hart and Lowell E. Staveland. 1988. Development of NASA-TLX (Task Load Index): Results of Empirical and Theoretical Research. In Human Mental Workload, Peter A. Hancock and Najmedin Meshkati (Eds.). Advances in Psychology, Vol. 52. North-Holland, 139 - 183. DOI : http://dx.doi.org/https: //doi.org/10.1016/S0166-4115(08)62386-9

13. Ke Huo, Vinayak, and Karthik Ramani. 2017. Window-Shaping: 3D Design Ideation by Creating on, Borrowing from, and Looking at the Physical World. In Proceedings of the Eleventh International Conference on Tangible, Embedded, and Embodied Interaction (TEI '17). ACM, New York, NY, USA, 37-45. DOI: http://dx.doi.org/10.1145/3024969.3024995

14. Johann Habakuk Israel, Laurence Mauderli, and Laurent Greslin. 2013. Mastering Digital Materiality in Immersive Modelling. In Proceedings of the International Symposium on Sketch-Based Interfaces and Modeling (SBIM '13). ACM, New York, NY, USA, 15-22. DOI : http://dx.doi .org/10.1145/2487381.2487388

15. Daniel F. Keefe, Robert C. Zeleznik, and David H. Laidlaw. 2007. Drawing on Air: Input Techniques for Controlled 3D Line Illustration. IEEE Transactions on Visualization and Computer Graphics 13, 5 (Sept 2007), 1067-1081. DOI :

http://dx.doi.org/10.1109/TVCG.2007.1060

16. Robert S. Kennedy, Norman E. Lane, Kevin S. Berbaum, and Michael G. Lilienthal. 1993. Simulator Sickness Questionnaire: An Enhanced Method for Quantifying Simulator Sickness. The International Journal of Aviation Psychology 3, 3 (1993), 203-220. DOI :

http://dx.doi.org/10.1207/s15327108ijap0303_3

17. Per Ola Kristensson and Shumin Zhai. 2004. SHARK2: A Large Vocabulary Shorthand Writing System for Pen-based Computers. In Proceedings of the 17th Annual ACM Symposium on User Interface Software and Technology (UIST '04). ACM, New York, NY, USA, 43-52. DOI : http://dx.doi .org/10.1145/1029632 .1029640

18. Tim McGraw, Esteban Garcia, and Drew Sumner. 2017. Interactive Swept Surface Modeling in Virtual Reality with Motion-tracked Controllers. In Proceedings of the Symposium on Sketch-Based Interfaces and Modeling (SBIM '17). ACM, New York, NY, USA, Article 4, 9 pages. DOI: http://dx.doi.org/10.1145/3092907.3092908

19. R. L. Potter, L. J. Weldon, and B. Shneiderman. 1988. Improving the Accuracy of Touch Screens: An Experimental Evaluation of Three Strategies. In Proceedings of the SIGCHI Conference on Human Factors in Computing Systems (CHI'88). ACM, New York, NY, USA, 27-32. DOI :

http://dx.doi.org/10.1145/57167.57171 
20. Ivan Poupyrev, Mark Billinghurst, Suzanne Weghorst, and Tadao Ichikawa. 1996. The Go-Go Interaction Technique: Non-linear Mapping for Direct Manipulation in VR. In Proceedings of the 9th Annual ACM Symposium on User Interface Software and Technology (UIST '96). ACM, New York, NY, USA, 79-80. DOI : http://dx.doi.org/10.1145/237091.237102

21. Ivan Poupyrev, Tadao Ichikawa, Suzanne Weghorst, and Mark Billinghurst. 1998. Egocentric Object Manipulation in Virtual Environments: Empirical Evaluation of Interaction Techniques. Computer Graphics Forum 17, 3 (1998), 41-52. DOI:

http://dx.doi.org/10.1111/1467-8659.00252

22. Falko Rheinberg, Regina Vollmeyer, and Stefan Engeser. 2003. Die Erfassung des Flow-Erlebens [The assessment of flow experience]. In Diagnostik von Selbstkonzept, Lernmotivation und Selbstregulation [Diagnosis of motivation and self-concept], J. Stiensmeier-Pelster and F. Rheinberg (Eds.). Hogrefe, Göttingen, 261-279.

23. Steven Schkolne, Michael Pruett, and Peter Schröder. 2001. Surface Drawing: Creating Organic 3D Shapes with the Hand and Tangible Tools. In Proceedings of the SIGCHI Conference on Human Factors in Computing Systems (CHI '01). ACM, New York, NY, USA, 261-268. DOI : http://dx.doi.org/10.1145/365024.365114

24. Ben Shneiderman. 1983. Direct Manipulation: A Step Beyond Programming Languages. Computer 16, 8 (Aug 1983), 57-69. DOI :

http://dx.doi.org/10.1109/MC. 1983.1654471
25. Ben Shneiderman and Catherine Plaisant. 2010. Designing the User Interface: Strategies for Effective Human-Computer Interaction (5th ed.). Addison-Wesley, Boston, USA.

26. Richard Stoakley, Matthew J. Conway, and Randy Pausch. 1995. Virtual Reality on a WIM: Interactive Worlds in Miniature. In Proceedings of the SIGCHI Conference on Human Factors in Computing Systems (CHI '95). ACM Press/Addison-Wesley Publishing Co., New York, NY, USA, 265-272. DOI : http://dx.doi.org/10.1145/223904.223938

27. Ivan E. Sutherland. 1963. Sketchpad: a Man-machine Graphical Communication System. In Proceedings of AFIPS'63. ACM, New York, NY, USA, 326-346. D0I : http://dx.doi.org/10.1145/800265.810742

28. Eva Wiese, Johann Habakuk Israel, Achim Meyer, and Sara Bongartz. 2010. Investigating the Learnability of Immersive Free-hand Sketching. In Proceedings of the Seventh Sketch-Based Interfaces and Modeling Symposium (SBIM '10). Eurographics Association, Aire-la-Ville, Switzerland, 135-142.

http://dl.acm.org/citation. cfm?id=1923363.1923387

29. Jacob O. Wobbrock, Andrew D. Wilson, and Yang Li. 2007. Gestures Without Libraries, Toolkits or Training: A \$1 Recognizer for User Interface Prototypes. In Proceedings of the 20th Annual ACM Symposium on User Interface Software and Technology (UIST '07). ACM, New York, NY, USA, 159-168. DOI :

http://dx.doi.org/10.1145/1294211.1294238 